\title{
Family-based association study of SELENBP1 in schizophrenia
}

\author{
Tetsufumi Kanazawa ${ }^{\mathrm{a}, \mathrm{b}}$, Stephen J. Glatt ${ }^{c}$, Stephen V. Faraone ${ }^{c}$, Hai-Gwo Hwu ${ }^{\text {d,e,f }}$, \\ Hiroshi Yoneda ${ }^{\mathrm{a}}$, Ming T. Tsuang ${ }^{\mathrm{b}, \mathrm{g}, \mathrm{h}, *}$ \\ a Department of Neuropsychiatry, Osaka Medical College, Osaka, Japan

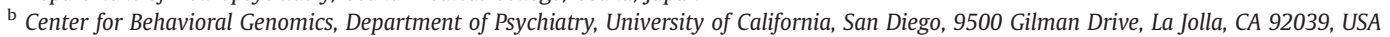 \\ ${ }^{\mathrm{c}}$ Department of Psychiatry and Behavioral Sciences, and Medical Genetics Research Center, SUNY Upstate Medical University, 750 East Adams Street, Syracuse, \\ NY, 13210, USA \\ d Department of Psychiatry, National Taiwan University Hospital and National Taiwan University College of Medicine, Taipei, Taiwan \\ e Institute of Epidemiology, College of Public Health, National Taiwan University, Taipei, Taiwan \\ ${ }^{\mathrm{f}}$ Department of Psychology, College of Science, National Taiwan University, Taipei, Taiwan \\ g Harvard Institute of Psychiatric Epidemiology and Genetics, Harvard Departments of Epidemiology and Psychiatry, 25 Shattuck Street, Boston, MA 02115, USA \\ ${ }^{\mathrm{h}}$ Veterans Affairs San Diego Healthcare System, 3350 La Jolla Village Drive, San Diego, CA 92161, USA
}

\section{A R T I C L E I N F O}

\section{Article history:}

Received 9 April 2009

Received in revised form 12 June 2009

Accepted 14 June 2009

\section{Keywords:}

Schizophrenia

SELENBP1

Selenium binding protein 1 gene

Family-based association study

Gene

\begin{abstract}
A B S T R A C T
The SELENBP1 gene previously was found to be up-regulated in microarray analysis of both peripheral blood cell and brain tissue samples from schizophrenia patients. Quantitative PCR analysis subsequently corroborated the altered expression of SELENBP1 in schizophrenia brain tissue samples from the Stanley Array Correction. The aim of this study was to extend those findings by employing family-based association methods to a sample of over 2400 individuals (including 1214 individuals affected by schizophrenia) of Han Chinese descent living in Taiwan. One of four haplotype-tagging SNPs and two different two-marker haplotypes showed nominally significant evidence for association with schizophrenia under an additive model, suggesting that genetic variation in SELENBP1 may influence risk for the disorder, while this significance did not remain when other inheritance models were considered. Further comprehensive analysis with other SNPs and haplotypes is needed and warranted.
\end{abstract}

(c) 2009 Elsevier B.V. All rights reserved.

\section{Introduction}

Schizophrenia is one of the most-often studied psychiatric illnesses, although we have not yet obtained clear insight into the etiology of the disorder. As technology develops in genetic research, a wide variety of methods have been employed in order to identify positional or functional candidate genes for this disorder (Faraone et al., 1999; Tsuang et al., 2005). We previously reported selenium binding protein 1 (SELENBP1) as a strong candidate gene based on data obtained through the use of cDNA microarray

\footnotetext{
* Corresponding author. Center for Behavioral Genomics, Department of Psychiatry, University of California, San Diego, Mail Code 0603, 9500 Gilman Drive, La Jolla, CA 92093, USA. Tel.: +1 858422 2464; fax: +1 8584222469.

E-mail address: mtsuang@ucsd.edu (M.T. Tsuang).
}

technology (Glatt et al., 2005). By comparing gene expression profiles of peripheral blood cells (PBCs) and postmortem brain tissue from two sets of schizophrenia patients and control subjects, we found that SELENBP1 was reliably up-regulated in both tissues in schizophrenia. In addition, immunohistochemical (IHC) and reverse transcriptase polymerase chain reaction (RTPCR) methods supported an upregulated expression of SELENBP1 in schizophrenic brains. The gene of interest is located on 1q21-22, which is strongly suggested as a linked region by Brzustowicz et al. (Brzustowicz et al., 2000); in addition, this region has been positively indicated by genome-scan meta-analysis in schizophrenia (Lewis et al., 2003). In our initial comparative study, SELENBP1 was one of just two genes (along with HLA-DRB1) that was significantly dysregulated in the same direction in both blood and brain in schizophrenia. SELENBP1 was up- 
regulated in both tissues while $H L A-D R B 1$ was downregulated. Considering that up-regulation of a transcript, it would be easier to verify than down-regulation, we selected first SELENBP1 for follow-up analyses, which included replication by QRT-PCR in a separate sample. Recently our group replicated significant up-regulation of SELENBP1 gene expression in brain tissue from schizophrenia patients in the Stanley Array Collection. Of note, the up-regulation of SELENBP1 was even stronger in a combined sample of brain tissue from schizophrenia patients and bipolar disorder patients with psychosis (Kanazawa et al., 2008). These findings have driven us to further analyze the relationship between this gene and schizophrenia.

The family-based association study method is somewhat classical compared to recent advanced methods such as genomewide case-control association analysis; however, family-based methods do have the advantage that they can avoid the possibility of type-I inferential errors due to population stratification (Gauderman et al., 1999). Presently, we have attempted to clarify the potential role of SELENBP1 in schizophrenia by determining if it shows patterns of association with the disorder in a large family-based association study. Briefly, our large sample (1214 affected individuals; total of 2408 samples; all of Taiwanese ancestry) had adequate power to detect an effect of each investigated single nucleotide polymorphism (SNP) with an odds ratio (OR) as low as 1.3 under conditions of optimal minor allele frequency (MAF between 0.15 and 0.25 ) and an OR as low as 1.5 under all MAFs greater than 0.05. To comprehensively survey the gene, we interrogated all four haplotype-tagging (ht) SNPs in SELENBP1. Overall, the aim of this study was to extend the previous gene expression findings for this gene in order to more clearly delineate the role of its protein in the etiology of the disorder.

\section{Methods}

\subsection{Sample recruitment}

All of the samples were obtained from the Taiwan Schizophrenia Linkage Study (TSLS). The TSLS program was designed to collect a large family sample from one ethnicity (Taiwan

Table 1

Descriptive statistics of the sample.

\begin{tabular}{lc}
\hline Statistic & $N$ (\% of Sample) \\
\hline Individuals & $2408(100)$ \\
Affection Status & \\
$\quad$ Affected & $1214(50.4)$ \\
Unaffected & $932(38.7)$ \\
Unknown & $262(10.9)$ \\
Sex & \\
Male & $1324(55.0)$ \\
Female & $1084(45.0)$ \\
Families & $616(100)$ \\
Affected per Family & \\
0 & $36(5.8)$ \\
1 & $43(7.0)$ \\
2 & $445(72.2)$ \\
3 & $88(14.3)$ \\
4 & $3(0.5)$ \\
5 & $1(0.2)$ \\
\hline
\end{tabular}

Han Chinese) in order to decrease the effects of complex ethnic composition. The analyzed participants in this study were comprised of 616 families with at least two siblings meeting DSM-IV criteria for schizophrenia or schizoaffective disorder (depressed type), including a total of 2408 members and 1214 schizophrenia patients (Table 1). All cases have a positive family history, as probands were ascertained as part of an affected sibling pair; however, some affected siblings did not contribute viable DNA samples and thus such families are listed as having only one affected offspring for the present analyses (although such families had at least two affected individuals in existence). The genotype data in 36 families without affected members were not utilized for determining the association analysis, but for constructing the LD block and for determining allele and haplotypes frequencies. More detailed information of clinical assessments are described in the previous paper (Hwu et al., 2005); briefly, the participants were screened using the Diagnostic Interview for Genetic Studies (DIGS) and Family Interview for Genetic Studies (FIGS), and diagnosed by two or more research psychiatrists. Clinical assessments were obtained by a research psychiatrist using medical records and a semi-structured interview that was based on DSM-IV. Informed consent was obtained from all participants, and all procedures involving human subjects were approved by the Institutional Review Boards of all project sites.

\subsection{Selection of SNPS}

Based on the data provided by the International Hapmap Project (http://www.hapmap.org), we selected tagging SNPs lying across the SELENBP1 gene, on the condition that the minor allele frequency was greater than 0.25 in the Han Chinese population. The selected four SNPs were rs2800953, rs10788804, rs744459, and rs2769264, all of which are located in intronic regions of the gene.

\subsection{Genotyping, data cleaning, and quality control}

Aliquots of 2408 high-quality DNA samples were sent from UCSD to the Harvard Partners Genotyping Facility at the Harvard Medical School-Partners Healthcare Center for Genetics and Genomics (HPCGG) for genotyping. Sequenom iPLEX technology was used for SNP genotyping at HPCGG using primers shown in Table 2. All genotyping procedures were executed per protocol (Oeth et al., 2005). Excluding the discordant genotypes ( $n=41$, $0.017 \%$ ), individual DNA samples were analyzed for HardyWeinberg equilibrium (HWE) as a final quality control. No individual marker out of the four SNPs showed significant deviation from expected frequencies.

\subsection{Data analysis}

Family-based association data were conducted using the PBAT algorithm (Lange et al., 2004) as implemented in the HelixTree Genetic Analysis Software suite, version 5.20 (GoldenHelix, Inc.; Bozeman, MT). The additive and dominant models were considered as the hereditary mode for all twomarker haplotypes comprised of adjacent markers, in which $p$ values were determined empirically through simulation using 1000 permutations of the data. For each individual SNP, 
Table 2

Details of primers.

\begin{tabular}{|c|c|c|c|}
\hline SNP_ID & Reverse $\mathrm{PCR}^{\mathrm{a}}$ & Forward PCR ${ }^{\mathrm{a}}$ & Extension primer \\
\hline rs2800953 & ACTTCTGCCTGAACCCTAAC & TGCCTAGCACAGACATTCAC & CCTAACTGGCCGTATTTAT \\
\hline rs10788804 & CTTAACAATGCTGCCTCCG & CTCTTCCTGCAATGTTTGCG & tGTGCAGAGTATAAGGAGG \\
\hline rs744459 & AGGCTGCTGGTTTAGGTCTG & TAGACCATAAGACACCTGCC & сTAGGTCTGGCTTCCTGCCC \\
\hline rs2769264 & TACCTCGCTGGAGTACAAAG & AAAACACCACTAGGTCCCTG & gTGGAGTACAAAGTTGGCCCA \\
\hline
\end{tabular}

${ }^{a}$ Both PCR primers contain a 10-base tail (ACGTTGGATG) for stability and to increase the mass of the PCR primers so that they do not show in the extension product mass window.

recessive and heterozygous advantage models were also tested. To correct for multiple testing, the Bonferroni correction was applied to exclude the possibility of type-I error rate at $5 \%$.

\section{Results}

Out of the four SNPs described previously, one SNP (rs2800953, allele A) in the 7th intron met a nominal significance level ( $p=0.034$, uncorrected) under the additive model. Moreover a two-marker haplotype including this SNP showed stronger significance in combination with the marker in the 9th intron (rs10788804, allele A). The $p$-value for haplotypes of rs10788804 (allele A) with rs2800953 was 0.004 (uncorrected) for allele A and 0.012 (uncorrected) for allele $\mathrm{G}$. The genomic organization and results under the additive model are shown in Fig. 1. Under the dominant model, the allele A for rs 10788804 combined with the A allele $(p=0.023$, uncorrected) and the $\mathrm{G}$ allele $(p=0.045$, uncorrected) of rs2800953 to form significantly risk-associated haplotypes. The statistical significance for two-marker haplotype combinations was basically independently calculated from a result of one marker. None of these results remained significant after correcting for multiple testing by the Bonferroni method. The critical $p$-value needed for signifi- cance was $0.05 / 16=0.003125$ for SNPs, whereas a value of $0.05 / 6=0.0083$ was required for haplotypes. Relative to the additive and dominant models, recessive and heterozygous models were not optimally powered in our sample, and so the results of these analyses were not considered further; however, the data are available upon request.

\section{Discussion}

The primary conclusion of this study is that our large familybased sample provides evidence for a considerable association between SELENBP1 polymorphisms and risk for schizophrenia; however, this conclusion must be tempered by the fact that none of the detected associations withstood correction for multiple testing. Yet, if some earlier indication of preference for a particular inheritance model were recognized, the two-marker haplotypes consisting of allele A for rs10788804 and allele A of rs2800953 may have remained significantly associated with the disorder even after correction for multiple testing. Power of our analyses was determined as follows: we set alpha $=0.05$, we modeled the specific family structures occurring in our sample, we tested additive and dominant models, we set the disease prevalence at 0.008 based on epidemiologic data from Taiwan, we set the evaluated marker as the disease marker, and we

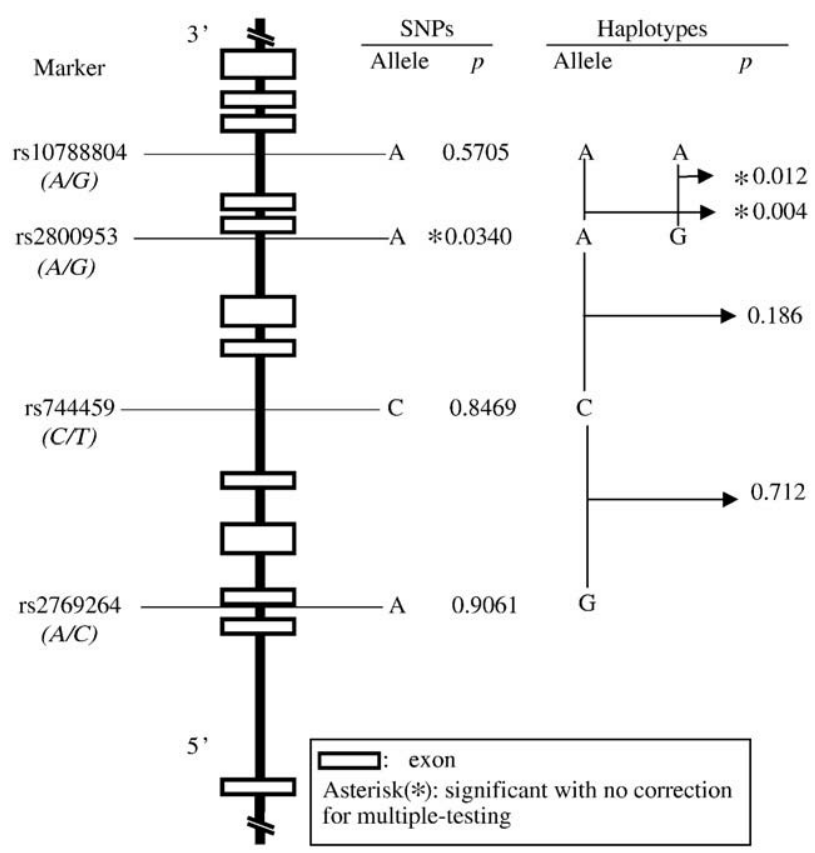

Fig. 1. Genomic organization and evidence for association with schizophrenia for SNPs and two-marker haplotypes of SELENBP1 (additive models). 
varied allele frequency from 0.05 to 0.45 in increments of 0.10 . At all minor allele frequencies at or above 0.05 , our sample provided $80 \%$ power or greater to detect a polymorphism or haplotypes with an associated odds ratio of 1.4 or greater under an additive model, while an allelic odds ratio of 1.2 or greater was detectable with the same level of power under a dominant model.

Our prior linkage analysis of this sample (Faraone et al. 2006) did not find substantial evidence for linkage in this particular region of chromosome 1q21-22; however, as the effect sizes of SELENBP1 variants we have detected presently are quite small, it is not expected that these would give rise to a significant linkage signal, nor is linkage in the region a prerequisite for association.

Overall, this study does identify clearly this is a gene worthy of further study. Future work on this gene should restrict the number of markers and models evaluated to increase the power to detect significant association, or select functional (rather than haplotype-tagging) polymorphisms which may be expected to have a larger effect size and smaller corresponding $p$-value.

Evidence has suggested that schizophrenia is a neurodevelopmental disorder (Harrison and Weinberger, 2005). In that scenario, the possible etiology of the disorder underlying the altered expression level of SELENBP1 is consistent because selenium plays a neuroprotective role against excitatory brain damage (Brauer and Savaskan, 2004). While the functional role of SELENBP1 has not yet been well established in the brain, selenium binding proteins (SBPs) have been shown to co-localize with g-actin at the growing tips of SY5Y neuroblastoma cells (Miyaguchi, 2004), which indicates the potential for SELENBP1 to be associated with the growth and remodeling of neurites. It is well-known that the trace mineral selenium is involved in a variety of metabolic processes, especially in antioxidant defenses (Schweizer et al., 2004). Selenium is incorporated into proteins to be transported by selenoproteins, which chaperone it to the brain through the blood-brain barrier (Richardson, 2005). On the other hand, selenium deficiency has been found to increase susceptibility to glutamate-induced neurotoxicity in rat hippocampus and even loss of hippocampal neurons (Savaskan et al., 2003). In addition to these in vitro studies, there is a report that schizophrenia patients treated with clozapine had significantly lower plasma selenium levels than patients treated with other medications (Vaddadi et al., 2003).

Thus, the increasing evidence provided by our group and others has suggested a potential role of SELENBP1 gene/ protein in the etiology of this disorder. To investigate this issue further, future work should focus on a certain genetic model and try to replicate the specific effects of our implicated SNPS and haplotypes, rather than taking the wide-ranging first-pass approach that we have taken which prevented us from declaring significance. At the same time it is warranted to explore the biological function of SELENBP1 gene in the human brain. To conclude, the aim of this study was to investigate whether a large family-based sample from one ethnicity provided clear evidence of association with SELENBP1 variants and schizophrenia, and we accomplished this goal in part by identifying several markers worthy of further analysis in large case-control and other family-based samples. In addition to replication of these specific markers, additional analyses of polymorphic sites in SELENBP1, including functional non-synonymous SNPs, seems warranted.

\section{Role of funding source}

This work was supported by a research project grant (1R01MH059624) from the United States National Institute of Mental Health (M.T.T.) and grants (90-8825PP and 91,92-9113PP) from the National Health Research Institute of Taiwan and the Genomic Medicine Research Program of Psychiatric Disorders of National Taiwan University Hospital, and partial support from Osaka Medical College, Neuropsychiatry Department. the NIMH and other grant providers had no further role in study design; in the collection, analysis and interpretation of data; in the writing of the report; and in the decision to submit the paper for publication.

\section{Contributors}

Author T. Kanazawa designed the study and wrote the first draft of the manuscript. Author S. J. Glatt designed the study, undertook the statistical analysis and revised the first draft. Author S. V. Faraone undertook the statistical analysis as same as Dr. Glatt. Author H. G. Hwu conducted the sample recruitment. Author Hiroshi Yoneda supervised the first author's work. Author Ming T. Tsuang managed the current work, and conducted the collaborative project. All authors contributed to and have approved the final manuscript.

\section{Conflict of interest}

The authors declare no conflict of interest on the current research.

\section{Acknowledgements}

The authors thank Allison Brown and Maura Regan at the HPCGG for coordinating and performing the genotyping, Frank Middleton and Jessica Lasky-Su at SUNY Upstate Medical University for assistance with detecting pedigree inconsistencies and Mendelian errors and statistical analyses, and Sharon Chandler at UCSD for technical assistance. The authors further thank our research and clinical collaborators in the Taiwan Schizophrenia Linkage Study Group, including Chih-Min Liu, Wei J. Chen, Ming-Ming Tsuang, ShihKai Liu, Ming-Hsien Shieh, Tzung-Jeng Hwang, Wen-Chen Ou-Yang, ChunYing Chen, Chwen-Cheng Chen, Jin-Jia Lin, Frank Huang-Chih Chou, ChingMo Chueh, Wei-Ming Liu, Mei-Hua Hall, Chiao- Chicy Chen, Jia-Jiu Lo, Jia-Fu Lee, Seng Shen, Yung Feng, Shin-Pin Lin, Shi- Chin Guo, Ming-Cheng Kuo, Liang-Jen Chuo, Chih-Pin Lu, Deng-Yi Chen, Huan-Kwang Ferng, Nan-Ying Chiu, Wen-Kun Chen, Tien-Cheng Lee, Hsin-Pei Tang, Yih-Dar Lee, Wu-Shih Wang, For-Wey Long, Tiao-Lai Huang, Jung-Kwang Wen, Cheng-Sheng Chen, Wen-Hsiang Huang, Shu-Yu Yang, and Cheng-Hsing Chen, and all of the colleagues at Osaka Medical College Neuropsychiatry Department especially Hiroki Kikuyama, Atsushi Tsutsumi, Masaki Nishiguchi, Akira Hokyo, Yoshiyuki Nishimoto (Ozone Hospital), and Jun Koh. Gratitude is expressed to Prof. Ian P. Everall, Gursharan Chana, and Brinda K. Rana for their great insight for this study. Finally, the authors thank the hospitals that participated in this study, including National Taiwan University Hospital and Medical College of National Taiwan University, National Taoyuan Psychiatric Center, National Tsaotun Psychiatric Center, National ChengKung University, Kai-Suan Psychiatric Hospital of Kaohsiung City, Yu-Li Veterans Hospital, and National Yu-Li Hospital.

\section{References}

Brauer, A.U., Savaskan, N.E., 2004. Molecular actions of selenium in the brain: neuroprotective mechanisms of an essential trace element. Reviews of Neuroscience 15, 19-32.

Brzustowicz, L.M., Hodgkinson, K.A., Chow, E.W., Honer, W.G., Bassett, A.S., 2000. Location of a major susceptibility locus for familial schizophrenia on chromosome 1q21-q22. Science 288, 678-872.

Faraone, S.V., Tsuang, D., Tsuang, M.T., 1999. Genetics of mental disorders: a guide for students, clinicians, and researchers. Guilford, New York, NY.

Faraone, S.V., Hwu, H.G., Liu, C.M., Chen, W.J., Tsuang, M.M., Liu, S.K., Shieh, M.H., Hwang, T.J., Ou-Yang, W.C., Chen, C.Y., Chen, C.C., Lin, J.J., Chou, F.H., Chueh, C. M., Liu, W.M., Hall, M.H., Su, J., Van Eerdewegh, P., Tsuang, M.T., 2006. Genome scan of Han Chinese schizophrenia families from Taiwan: confirmation of linkage to 10q22.3. American Journal of Psychiatry 163, 1760-1766.

Gauderman, W.J., Witte, J.S., Thomas, D.C., 1999. Family-based association studies. Journal of the National Cancer Institute Monographs 31-37. 
Glatt, S.J., Everall, I.P., Kremen, W.S., Corbeil, J., Sasik, R., Khanlou, N., Han, M, Liew, C.C., Tsuang, M.T., 2005. Comparative gene expression analysis of blood and brain provides concurrent validation of SELENBP1 up-regulation in schizophrenia. Proceedings of the National Academy of Sciences of the United States of America 102, 15533-15538.

Harrison, P.J., Weinberger, D.R., 2005. Schizophrenia genes, gene expression, and neuropathology: on the matter of their convergence. Molecular Psychiatry 10, 40-68 [image 45].

Hwu, H.G., Faraone, S.V., Liu, C.M., Chen, W.J., Liu, S.K., Shieh, M.H., Hwang, T.J., Tsuang, M.M., OuYang, W.C., Chen, C.Y., Chen, C.C., Lin, J.J., Chou, F.H., Chueh, C.M., Liu, W.M., Hall, M.H., Tsuang, M.T., 2005. Taiwan schizophrenia linkage study: the field study. American Journal of Medical Genetics B Neuropsychiatric Genetics 134, 30-36.

Kanazawa, T., Chana, G., Glatt, S.J., Mizuno, H, Masliah, E., Yoneda, H., Tsuang, M.T, Everall, I.P., 2008. The utility of SELENBP1 gene expression as a biomarker for major psychotic disorders: replication in schizophrenia and extension to bipolar disorder with psychosis. American Journal of Medical Genetics B Neuropsychiatric Genetics 147B (6), 689-689.

Lange, C., DeMeo, D., Silverman, E.K., Weiss, S.T., Laird, N.M., 2004. PBAT: tools for family-based association studies. American Journal of Human Genetics 74, 367-369.

Lewis, C.M., Levinson, D.F., Wise, L.H., DeLisi, L.E., Straub, R.E., Hovatta, I. Williams, N.M., Schwab, S.G., Pulver, A.E., Faraone, S.V., Brzustowicz, L.M., Kaufmann, C.A., Garver, D.L., Gurling, H.M., Lindholm, E., Coon, H., Moises, H.W., Byerley, W., Shaw, S.H., Mesen, A., Sherrington, R., O'Neill, F.A., Walsh, D., Kendler, K.S., Ekelund, J., Paunio, T., Lönnqvist, J., Peltonen, L. O'Donovan, M.C., Owen, M.J., Wildenauer, D.B., Maier, W., Nestadt, G., Blouin, J.L., Antonarakis, S.E., Mowry, B.J., Silverman, J.M., Crowe, R.R., Cloninger, C.R., Tsuang, M.T., Malaspina, D., Harkavy-Friedman, J.M.,
Svrakic, D.M., Bassett, A.S., Holcomb, J., Kalsi, G., McQuillin, A., Brynjolfson, J., Sigmundsson, T., Petursson, H., Jazin, E., Zoëga, T., Helgason, T., 2003. Genome scan meta-analysis of schizophrenia and bipolar disorder, part II: schizophrenia. American Journal of Human Genetics 73, 34-48.

Miyaguchi, K., 2004. Localization of selenium-binding protein at the tips of rapidly extending protrusions. Histochemistry and Cell Biology 121, 371-376.

Oeth, P., Beaulieu, M., Park, C., Kosman, D., del Mistro, G., van den Boom, D., Jurinke, C., 2005. iPLEX ${ }^{\mathrm{TM}}$ assay: increased plexing efficiency and flexibility for MassARRAY system through single base primer extension with massmodified terminators. City, pp. 1-12.

Richardson, D.R., 2005. More roles for selenoprotein P: local selenium storage and recycling protein in the brain. Biochemical Journal 386, e5-e7.

Savaskan, N.E., Brauer, A.U., Kuhbacher, M., Eyupoglu, I.Y., Kyriakopoulos, A., Ninnemann, O., Behne, D., Nitsch, R., 2003. Selenium deficiency increases susceptibility to glutamate-induced excitotoxicity. FASEB Journal 17, $112-114$.

Schweizer, U., Brauer, A.U., Kohrle, J., Nitsch, R., Savaskan, N.E., 2004. Selenium and brain function: a poorly recognized liaison. Brain Research Brain Research Reviews 45, 164-178.

Tsuang, M.T., Nossova, N., Yager, T., Tsuang, M.M., Guo, S.C., Shyu, K.G., Glatt, S.J., Liew, C.C., 2005. Assessing the validity of blood-based gene expression profiles for the classification of schizophrenia and bipolar disorder: a preliminary report. American Journal of Medical Genetics B Neuropsychiatric Genetics 133, 1-5.

Vaddadi, K.S., Soosai, E., Vaddadi, G., 2003. Low blood selenium concentrations in schizophrenic patients on clozapine. British Journal of Clinical Pharmacology 55, 307-309. 